\title{
Size Optimization of Microsatellite Primary Structure
}

\author{
Ning Wang ${ }^{1, \text { a }}$, Yong Zhao ${ }^{1, b^{*}}$,Wen Yao ${ }^{1, \mathrm{c}}$ and Yi Zhang ${ }^{1, \mathrm{~d}}$ \\ ${ }^{1}$ College of Aerospace Science and Engineering, \\ National University of Defense Technology, \\ Changsha 410073, Hunan, China \\ aemail: 15570866341@163.com, bemai: zhaoyong@nudt.edu.cn \\ cemail: yaowen@nudt.edu.cn, demail: gfkdzhangyi@126.com
}

\begin{abstract}
Keywords: Structural Size Optimization, CAD/CAE, Microsatellite Design
Abstract: This paper focuses on structural size optimization. The optimization problem of "TianTuo-3" microsatellite, which is successfully launched in September 20, 2016, is formulated mathematically. The most character of the problem is that outcomes under certain inputs are analyzed by commercial software, rather than analytical formulations. Solving the problem could be computationally unaffordable. Two kinds of algorithms, gradient-based and intelligent, are applied and compared in effect and efficiency. Results show that although both algorithms of NLPQL and ASA get a good effect, with total mass reduced $33.1 \%$ for the former and $32.7 \%$ the latter, NLPQL is more efficient, with less iterations (nearly half of ASA). It can be concluded that nonlinearity of the objective function and constraints are not strong and gradient-based algorithms are qualified to the problem with high efficiency.
\end{abstract}

\section{Introduction}

With the improvement of computing capacity, computational models play an increasing role as predictive models for complex engineering systems. Techniques of Computer Aided Design (CAD) and Computer Aided Engineering (CAE) are essential tools to construct computational models of structure. CAD makes it easy to present design results in a visual manner and convenient to change the designing scheme. CAE helps verify the designing scheme in computer, minimizing or even eliminating the use of physical prototypes. They both contribute to reducing cost and shortening development period, which are main purposes of modern product design.

Many engineering problems involve structural optimization, to satisfy certain requirements (e.g. limit state conditions) while minimizing certain quantities (e.g. resources spent) and maximizing others (e.g. structural safety) [1]. The requirements to satisfy are called constraints and the functions to minimize/maximize are called objective functions. Optimization problems can be classified in three categories [2]:

(1) Size optimization problems, which typically consist of the calibration of the cross sectional properties and dimensions of the structural elements.

(2) Shape optimization problems, which consist of the optimization of the boundary shape, generally used to reduce stresses or to make the stress distribution uniform with no increase of structural weight.

(3) Topology optimization problems, in which the goal is to determine the optimal distribution of material, given the design space, boundary conditions, loads and required design performance.

Topology optimization is in conceptual design process and shape \& size optimization are in detailed design process. Examples of the three categories are shown in Fig. 1.

Solving structural optimization problem by integrating CAD/CAE is an effective method [3]. F.Gasser [4] systematically illustrates application of FEA in satellite design, including static and dynamic response analysis, failure analysis, and machining simulation. K. Suresh [5] constructs a precise FE-model of a microsatellite by MSC PARTRAN and calculates the natural frequency by MSC NASTRAN, whose discrepancy with real test is limited to 12\%. H. S. Park and X.P. Dang [6] proposed a structural optimization framework based on CAD/CAE integration and meta-modeling techniques, successfully applied in a numeral example. In addition, various structural optimization 
tools, based on idea of integration, are developed, e.g. Multidisciplinary Computing Environment for Aeromechanic (MDICEAE) by America Fluid Dynamics Research Center (FDRC), Integrated Hypersonic Aeromechanic Tool (IHAT) by America Navy [7] and Multidisciplinary Conceptual Design (MCDesign) by Northwestern Poly-technical University, etc. Even so, applications in practical engineering are still limited, because optimization problem using high-fidelity model is often calculation unaffordable. For example, one medium scale FE-analysis in engineering may cost hours, not to say hundreds of iterations in optimization. So, choosing a proper optimization algorithm, achieving a balance between effect and efficiency, is very important.

This paper focuses on structural size optimization. The optimization problem of "TianTuo-3" microsatellite is formulated mathematically in Section 2. To solve the calculation-consuming problem, the framework of method, including techniques of geometry parameterization and automatic re-meshing, is discussed in Section 3. Section 4 is the solution to the problem, discussing the better optimization algorithm. Conclusions are summarized in the last section (Section 5).

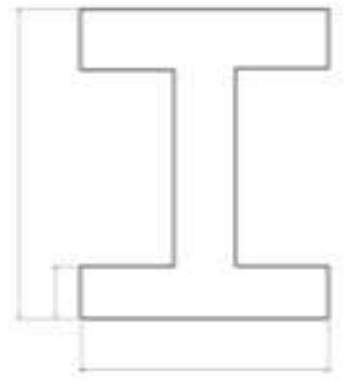

(a)

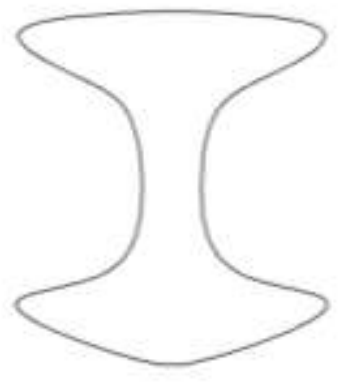

(b)

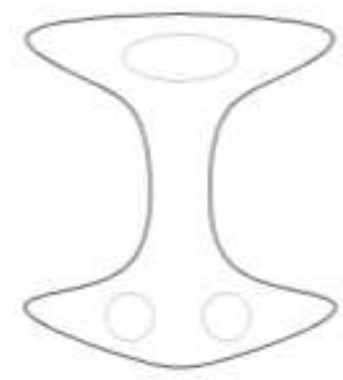

(c)

Fig. 1 Examples of structural optimization: (a) sizing, (b) shape, and (c) topology

\section{The optimization model}

During the structure design of microsatellite TT-3, which is successfully launched in September 20, 2016, sizes of primary structure (Fig. 2) are hard to determine. $t_{f}$ and $t_{r}$ are thickness of main frame and inner ribs, and $h_{r}$ stands for height of the ribs. Based on designer' experience, the larger $t_{f}, t_{r}$, and $h_{r}$ are, the safer the primary structure is. However, large thicknesses and height means big mass, which will add to manufacturing and launching cost. To balance the total structure mass and stress requirement, the design problem can be formulated as the following optimization problem:

$$
\begin{aligned}
& \text { minimize } f\left(t_{f}, t_{r}, h_{r}\right) \\
& \text { subject to } g_{i}\left(t_{f}, t_{r}, h_{r}\right) \leq n_{i}(i=1,2, \cdots)
\end{aligned}
$$

where $f\left(t_{f}, t_{r}, h_{r}\right)$ is the total mass of primary structure, which can be calculated when the three sizes and structure materials are determined. As with $g_{i}\left(t_{f}, t_{r}, h_{r}\right)$, three basic requirements are discussed in the following.

Considering that main purpose of structure is to provide enough strength and stiffness to resist static and dynamic loads, stress should be limited in a certain level under overload condition when launching. The upper limit is determined by character of material and how reliable we want the design, which is described by safety factor. The constraint can be expressed by:

$$
s \leq[\sigma]=\frac{\sigma_{g}}{N}
$$

where $s$ is the max stress value of whole primary structure, and $[\sigma]$ is allowable stress, which is calculated by $\sigma_{s}$ (yield stress of selected material) and $N$ (safety factor).

Additionally, enough stiffness is also important. There are many precise payloads attached to the primary structure, and they are sensitive to displacement, e.g. the attitude control system could lose 
efficacy if the position of momentum wheels changed. Besides, a big displacement can also cause problem of equipment interference and collision, resulting in reduced service life, and even cause a disaster. Considering momentum wheel is precise payload in "TT-3", the displacement in position where momentum wheel attached to should be limited:

$$
u \leq[u]
$$

where $u$ stands for the displacement, and $[u]$ is corresponding maximum displacement permitted.

Finally, resonance should be avoided. Under complex dynamic loads during launching, it is probable to provoke a frequency of the structure, which seriously deteriorates structure stability. So the first natural frequency of primary structure $f$ should be far away from validation frequency $f_{0}$ caused by the vehicle:

$$
f \geq f_{0}
$$

Based on design requirements and engineering practice, $N=2,[u]=5 \mathrm{~mm}$, and $f_{0}=20 \mathrm{HZ}$. Launching overload is about $10 \mathrm{~g}$ in three axis directions. Considering manufacturing, $t_{f} \in[2,7]$, $t_{r} \in[1,6]$, and $h_{r} \in[30,70]$ (Units: $\mathrm{mm}$ ).

Although the optimization problem has been expressed in mathematic formulations, to solve the problem is still difficult. The calculation cost is high because there are no analytical formulations between inputs and outputs. Besides, multi-constraints add to difficulties in searching the optimal design. So choosing an efficient optimization algorithm is challenging.

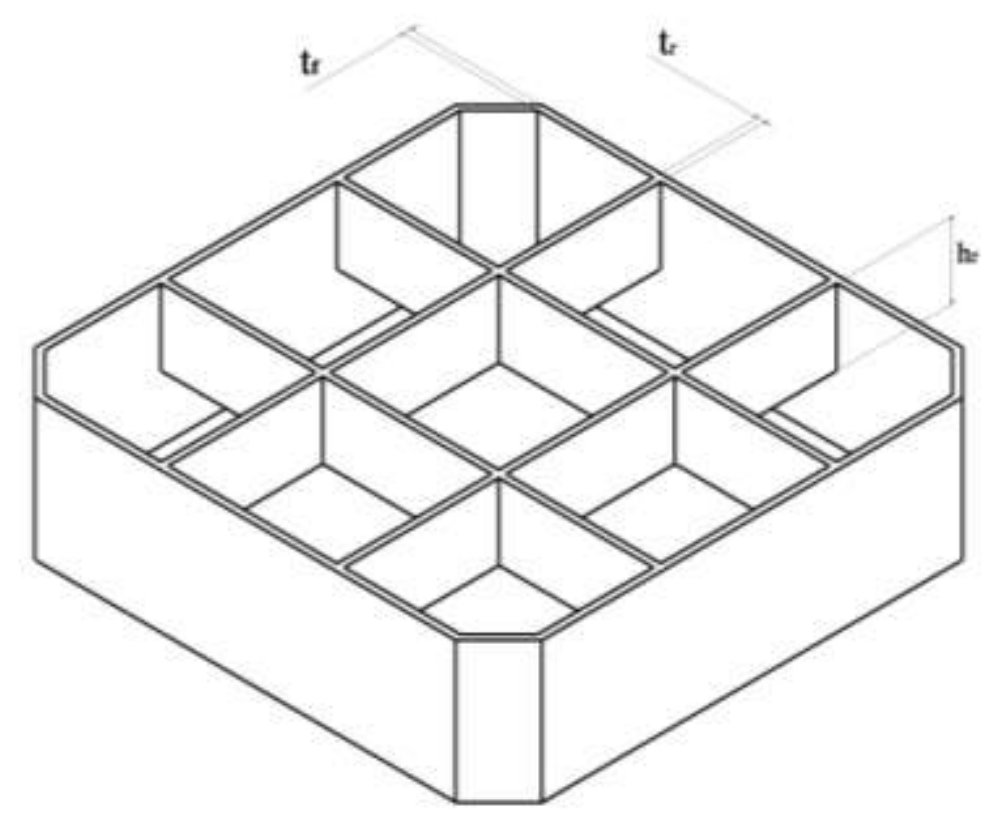

Fig. 2 Sizes of primary structure

\section{The framework}

When design changes, the 3-D model and FE-model should be updated automatically. Techniques of geometry parameterization and automatic re-meshing are able to solve the problem. Parameterization is not just mapping certain parameters to model sizes. Clarifying relationships of sizes and constructing necessary constraints are also important for getting a correct updated 3-D model without geometry clash and interference. After geometry updating, automatic re-meshing technique is required. When dealing with simple structure, macro commands of software are competent to re-mesh geometry automatically, but when it comes to complex structure, previous method is no longer feasible, resulting in bad meshes, which may causes error in following calculation. Measures 
should be taken to prevent such cases. The optimization framework is shown in Fig. 3. Techniques of geometry parameterization and automatic re-meshing using commercial software are discussed in the following.

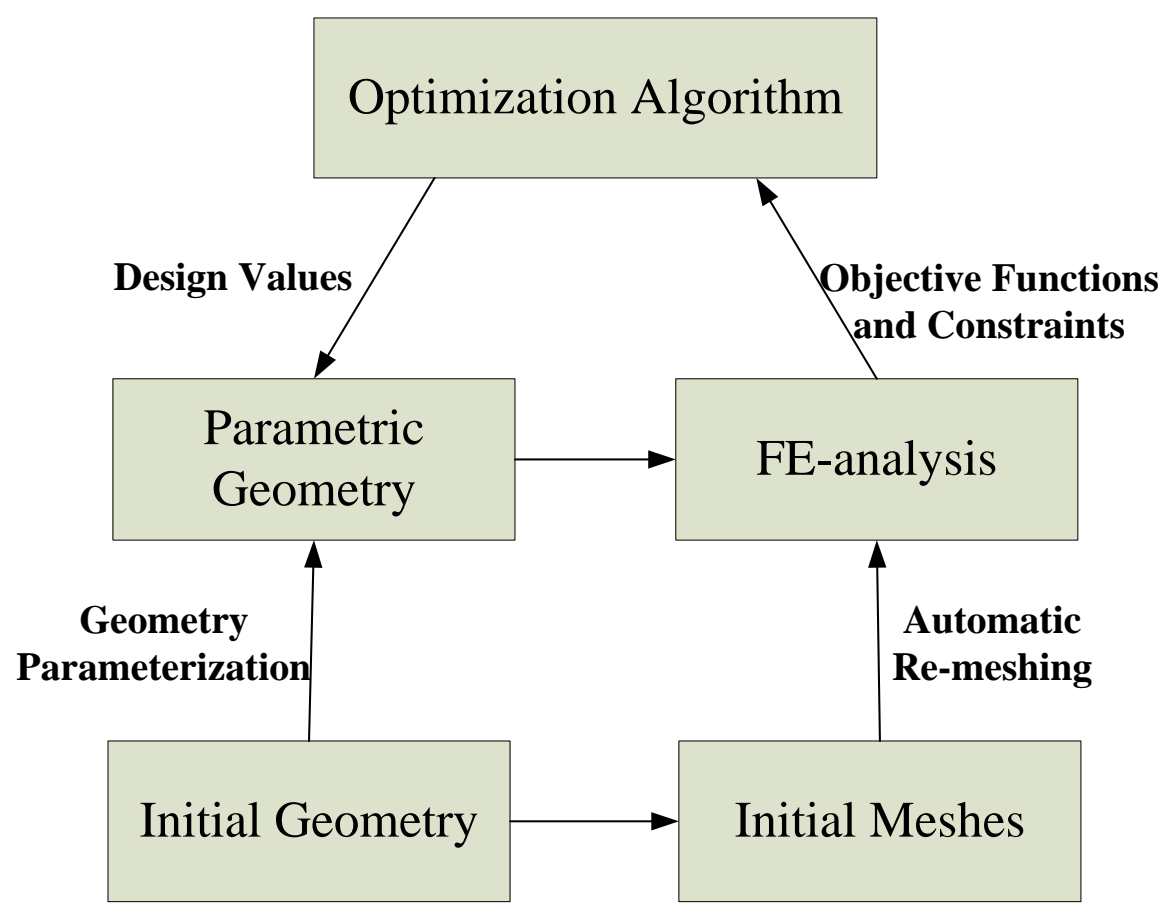

Fig. 3 The optimization framework

Geometry parameterization. CATIA is a powerful multifunction platform for product design, widely used in various field, particularly in aerospace. 3-D modeling function of the software is applied here to getting a parametric geometry. First, parameters of initial geometry are defined to represent design values. Second, the initial geometry is constructed by sketches and properties. During the drafting of sketches, constraints are defined to limit sizes, positions and relationships of geometrical elements so that the whole configuration can update without error. Under-constraint and over-constraint can both cause error. Third, parameters defined in the first step are mapped to sketches and properties, e.g. length of a line in sketch or height of stretching property. Finally, the parameters are linked to external document, so that changing the document can drive model updating, where CATIA macro commands are used to guarantee the automation of the process.

Automatic re-meshing technique. ABAQUS is applied to conduct FE-analysis. After the updated geometry imported, material properties, boundary conditions and loads should be defined. Then, re-meshing is required. For complex structures, adding number of intimal seeds and cutting geometry into simple parts are both effective measures to overcome the problem caused by using macro commands to re-mesh. Examples are shown in Fig. 4.

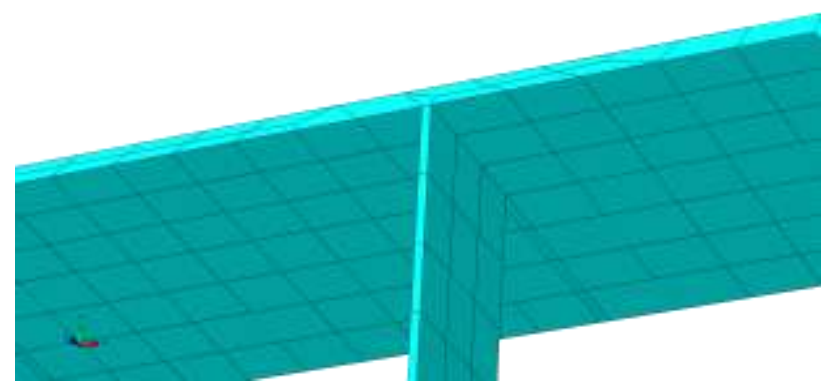

(a) Automatic re-meshing without partition

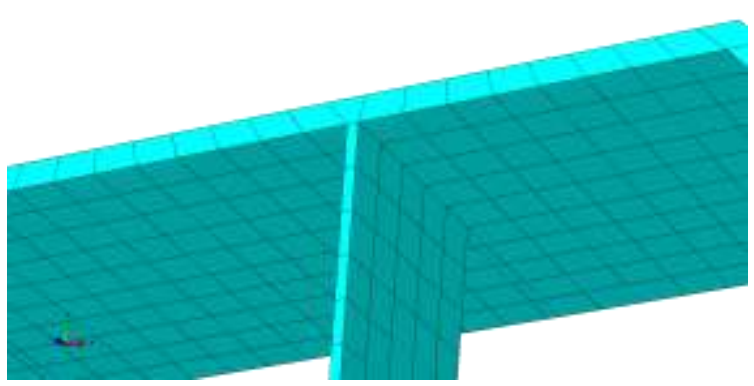

(b) Automatic re-meshing with partition

Fig. 4 Bad (a) and modified (b) meshes 
Integration of CATIA and ABAQUS. ISIGHT is a multidisciplinary computing environment with comprehensive optimization strategies and specified interfaces to various kinds of commercial software, including CATIA and ABAQUS. Also, integration can be achieved by invoking Windows BAT commands if there are no specified interfaces for uncommonly used software. There are many kinds of optimization algorithm, gradient-based and intelligent, e.g. NLPQL and ASA. Different algorithms are suitable for different problems. Choosing a proper algorithm is a coordinate issue, considering calculating efficiency, accuracy and robustness.

\section{Results}

The initial FE-analysis. After the initial geometry of primary structure imported to ABAQUS, material should be assigned. 6061 aluminium alloy is chosen and its properties are listed in Table 1 . To simulate the forces during launching, lumped mass points, representing power, sub-satellite and four momentum wheels, are created and attached to the ribs by means of MPC constraints. Masses of these components are listed in Table 2 and positions are the same as practice (shown in Fig. 5). Four corners of the primary structure are fixed and "gravity" load is applied to the whole space with 10g in three axis directions. When meshing, partitions of frame and ribs are necessary, the reason of which is explained in Section 3. 2060 hex-shaped elements are got by meshing technique of "sweep". Mesh verifying shows there are no error meshes, indicating calculation could be conducted next. The calculated displacements of the structure are shown in Fig. 6, maximum of which is $1.17 \mathrm{~mm}$ in the most red region. Total mass of the structure, is listed in Table 3.

Table 1 Properties of 6061 aluminium alloy

\begin{tabular}{cccc}
\hline $\begin{array}{c}\text { Density } \\
{\left[\mathrm{kg} / \mathrm{m}^{3}\right]}\end{array}$ & $\begin{array}{c}\text { Modulus of Elasticity } \\
{[\mathrm{GPa}]}\end{array}$ & $\begin{array}{c}\text { Yield Stress } \\
{[\mathrm{MPa}]}\end{array}$ & Poisson' ratio \\
\hline 2690 & 68.9 & 276 & 0.33 \\
\hline & Table 2 Masses of attached components $[\mathrm{kg}]$ \\
\hline Power & Sub-satellite & Momentum Wheels ( 4) \\
\hline 0.65 & 1 & 0.27 \\
\hline
\end{tabular}

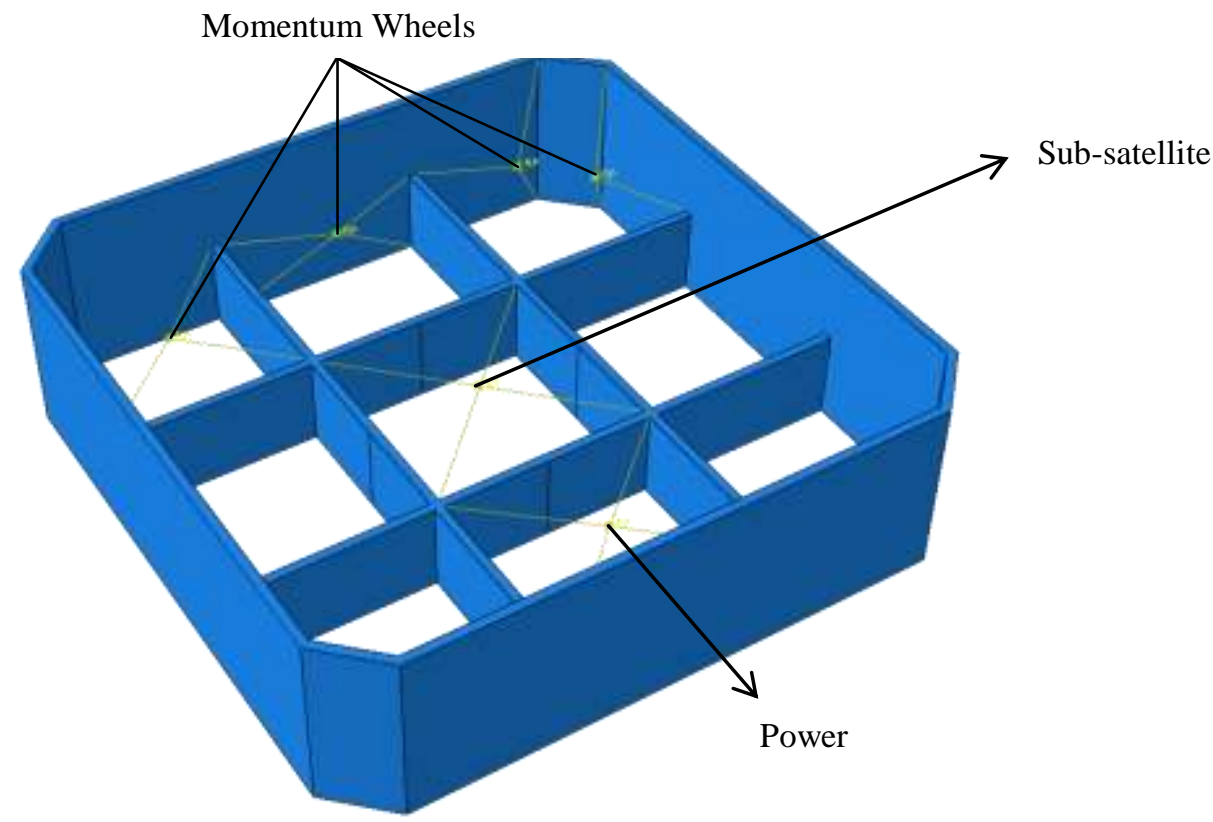

Fig. 5 Position of attached components 


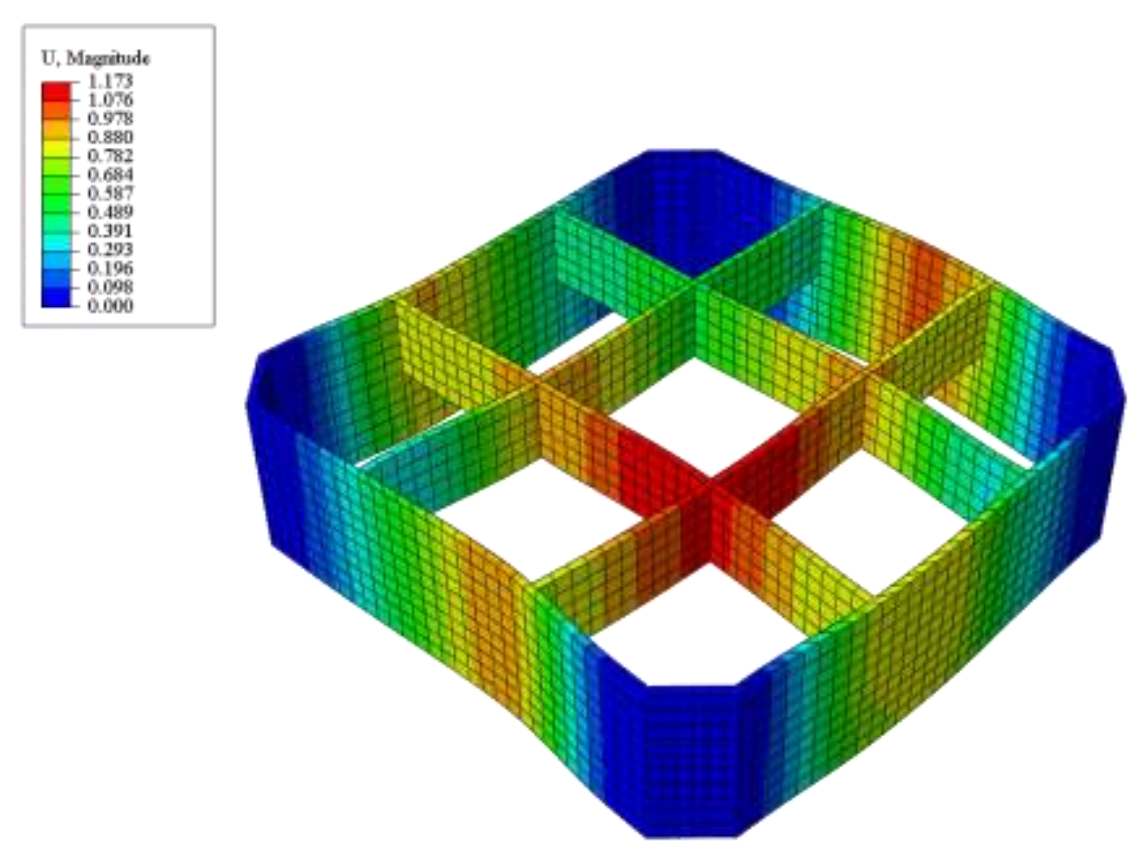

Fig. 6 Displacement of the initial primary structure

Optimization algorithms. This is a multi-constraints and nonlinear optimization problem. The most important character of the problem is that relations between design values and objective function $\&$ constraints are not explicit, only available by calculation-consuming FE-analysis. So, an efficient algorithm, with iterations as little as possible, is needed. There are 16 kinds of algorithms in ISIGHT, including gradient-based ones, e.g. MMFD, NLPQL, etc., intelligent ones, e.g. ASA, GA, etc., and combinatorial ones, e.g. Pointer. How to choose an effect and efficient algorithm is a challenging but important work. Because the objective function (total mass) is monotonically increasing with all the three inputs, gradient-based algorithm will find the minimal mass quickly. To make a comparison, two kinds of frequently-used algorithms, NLPQL and ASA, are applied, and total mass with the increase of iterations is shown in Fig. 7. Initial and optimal designs by NLPQL and ASA are listed in Table 3. From the table, better designs are achieved, with mass reduced $33.1 \%$ for NLPQL and $32.7 \%$ for ASA. Comparing the two algorithms, although minimum masses are basically the same, NLPQL consume less calculating resources than ASA, whose iteration number is $43 \%$ of ASA. The result is in accord with previous judgment.

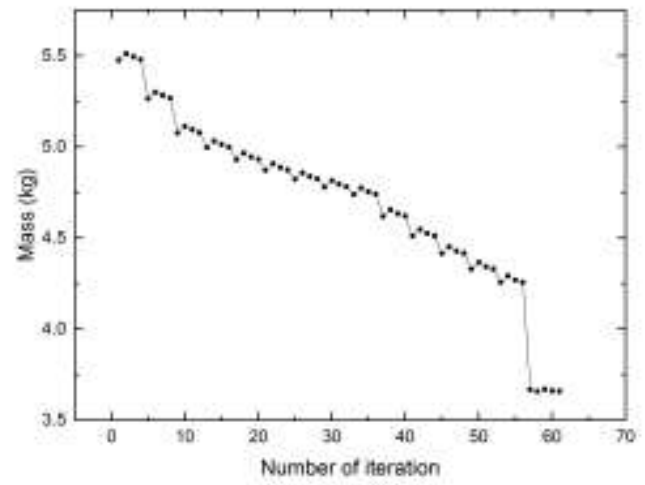

(a) NLPQL

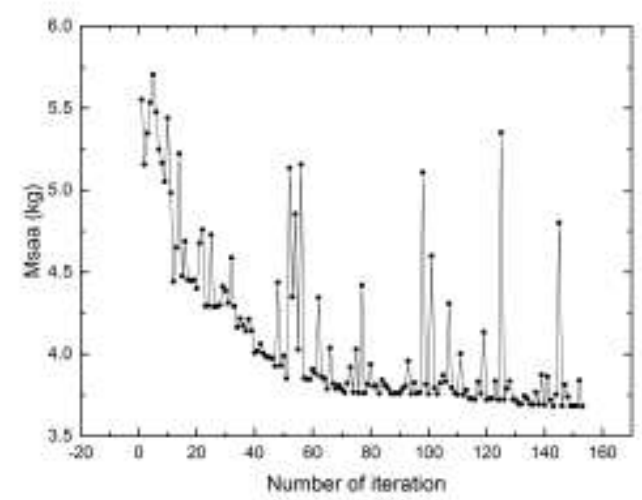

(b) ASA

Fig. 7 Mass with the number of iterations for different algorithm: (a) NLPQL and (b) ASA 
Table 3 Comparison of optimal designs by NLPQL and ASA with initial design

\begin{tabular}{cccccc}
\hline & $\boldsymbol{t}_{\boldsymbol{f}}[\mathrm{mm}]$ & $\boldsymbol{t}_{\boldsymbol{r}}[\mathrm{mm}]$ & $\boldsymbol{h}_{\boldsymbol{r}}[\mathrm{mm}]$ & Mass $[\mathrm{kg}]$ & $\begin{array}{c}\text { Iteration } \\
\text { numbers }\end{array}$ \\
\hline Initial & 5 & 5 & 50 & 5.48 & - \\
NLPQL & 2 & 1.8 & 30 & 3.67 & 78 \\
ASA & 2 & 1.7 & 34 & 3.69 & 179 \\
\hline
\end{tabular}

\section{Conclusions}

This paper demonstrates how to solve a microsatellite structural optimization problem by integrating $\mathrm{CAD} / \mathrm{CAE}$. Conclusions are summarized in the following:

(1) Parameterization is not just mapping certain parameters to model sizes. Clarifying relationships of sizes and constructing necessary constraints are also important for getting a correct updated 3-D model without geometry clash and interference.

(2) Adding number of intimal seeds and cutting geometry into simple parts are effective measures to overcome the bad-meshes problem when applying automatic re-meshing technique to complex geometry.

(3) Optimal designs, with mass reduced $33.1 \%$ for NLPQL and $32.7 \%$ for ASA, are achieved, getting a good optimization results.

(4) Because nonlinearity of the objective function and constraints are not strong, gradient-based algorithms are qualified to the problem with a high efficiency.

\section{References}

[1] P. Y. Papalambros and D. J. Wilde, Principles of optimal design: modeling and computation, 2nd ed., Cambridge University Press, Cambridge, UK, 2000.

[2] M. B. Marques, Timo Pripler, and M. R. Tiago Arruda, Structural optimization using the finite element method, Universidade Católica Portuguesa Faculdade de Engenharia, 2014.

[3] S. H. Lee, A CAD/CAE integration approach using feature-based multi-resolution and multi-abstraction modelling techniques, Computer-Aided Design, 2005, 37(9): 941-955.

[4] F. Gasser, Finite element analysis for satellite structures. Springer London Heidelberg New York Dordrecht. ISBN 978-1-447114637-7(eBook).

[5] K.Suresh, Design, analysis and development of micro-satellite structure, Faculty of Mechanical Engineering Anna University, Chennai, 2009.

[6] H. S. Park, X.P. Dang, Structural optimization based on CAD/CAE integration and metamodeling techniques, Computer-Aided Design, 2010, 42(10): 889-902.

[7] M. L. Baker, M. L. Munson, and G. W. Hoppus, The inntegrated hypersonic aeromechanics tool(IHAT)buuild 4, 10th AIAA/ISSMO Multidisciplinary Analysis and Qptimization Conference, Albany, New York. AIAA 2004-4565, 2004. 